\title{
Comparison Between Pyogenic Postoperative and Native Vertebral Osteomyelitis : Clinical Features, Curative Effect and Analysis of Prognostic Factors
}

maimaitiaili abudurexiti

First Affiliated Hospital of Xinjiang Medical University

maierdan maimaiti ( $\nabla$ mardanmmtmx@163.com )

First Affiliated Hospital of Xinjiang Medical University

Mulade Maierdan

Tongji University

Tao Xu

First Affiliated Hospital of Xinjiang Medical University

xieraili saimaiti

First Affiliated Hospital of Xinjiang Medical University

Wei Bing Sheng

First Affiliated Hospital of Xinjiang Medical University

Yang Zhou

First Affiliated Hospital of Xinjiang Medical University

\section{Research Article}

Keywords: Vertebral Osteomyelitis, Spinal Surgery, Suppuration, Outcome

Posted Date: January 4th, 2021

DOI: https://doi.org/10.21203/rs.3.rs-133906/v1

License: (c) (i) This work is licensed under a Creative Commons Attribution 4.0 International License.

Read Full License 


\title{
Comparison between Pyogenic Postoperative and Native Vertebral Osteomyelitis : Clinical features, curative effect and analysis of prognostic factors
}

\author{
Maierdan Maimaiti [1]mailto:mardanmmtmx@163.com , Maimaitiaili Abudurexiti ${ }^{[1]}$, Mulade Maierdan \\ ${ }^{[2]}, \mathrm{Tao}_{\mathrm{Xu}}{ }^{[3]}, \mathrm{Xieraili} \mathrm{Saimaiti}^{[4]}$, Wei-Bing Sheng ${ }^{[5]}$, Yang Zhou ${ }^{[6]}$ \\ 1. 3, 4, 5,6 Department of spine surgery, First Affiliated Hospital of Xinjiang Medical University, \\ Urumqi 830054, Xinjiang Uyghur Autonomous Region, China. \\ ${ }^{2}$ Grade 2018 in clinical Medicine of Tongji University Medical School, Shanghai 200092, \\ China.
}

Abstract

\section{Background}

Pyogenic spondylodiscitis(PS) is a potentially life-threatening infection burdened with high morbidity rates. Despite the rising incidence, the proper diagnosis and treatment of PS are still controversial. Postoperative Vertebral Osteomyelitis(PVO) is a clinical challenge, for there were few reports about the treatment results of PVO before, and further more few studies have compared PVO with native vertebral osteomyelitis(NVO). The purpose of this study was to compare and describe the microbiology, clinical characteristics, treatment and curative effect between PVO and NVO, and analyze the prognostic factors as well.

\section{Methods}

The clinical data of $5^{2}$ patients with pyogenic spondylitis admitted to the Spine Surgery Department of the First Affiliated Hospital of Xinjiang Medical University from January 2010 to December 2019 were retrospectively analyzed. There were 30 patients in native vertebral osteomyelitis (NVO) group, including 18 males and 12 females, with an average age of $50.47 \pm$ 20.45 years old (aged from 15 to 73); 22 patients in postoperative vertebral osteomyelitis (PVO) group, including 13 males and 9 females, with an average age of $51.45 \pm 16.97$ years old (aged from 14 to 73 ). In Group NVO, 23 cases (76.7\%) were located in lumbar vertebrae, 5 cases (16.7\%) in thoracic vertebrae and 2 cases (6.7\%) in cervical vertebrae; in Group PVO, 16 cases $(72.7 \%)$ in lumbar vertebrae and 6 cases (27.3\%) in thoracic vertebrae. 29 patients had 
had neurological dysfunction before surgery was taken. There were 26 cases of grade D (16 cases in Group NVO and 10 cases in Group PVO) and 3 cases of grade C (1 case in Group NVO and 2 cases in Group PVO), following the instructions of American Spinal Injury Association (Asia) neurological function classification. All patients were given bed rest, nutritional support and antibiotic therapy; surgical treatment for patients with poor outcomes or aggravated symptoms. Patients were followed up at 1, 3, 6 and 12 months after surgery, including leukocyte count, ESR and CRP, X-ray, CT three-dimensional reconstruction and MRI were performed. The changes of visual analogue scale (VAS) and Asia neurological function classification were observed to evaluate the clinical efficacy simultaneously.

\section{Results}

All patients were followed up for 12-24 months. Till the last follow-up, 3 patients in Group NVO recurred, the recurrence rate was 10\% (3 / 30), 9 patients in Group PVO recurred, the recurrence rate was 40.1\% (9 / 22), the recurrence rate of Group PVO was higher than that of Group NVO, the difference was statistically significant $(\mathrm{P}=0.009)$. Both groups were treated with intravenous and oral antibiotics, and the time of antibiotic treatment in Group PVO was longer than that in Group NVO, however the difference was not statistically significant $(\mathrm{P}=0.094, \mathrm{P}=0.062)$. Among 44 patients with spinal internal fixation, $13.6 \%$ (1 NVO, 5 PVO) had recurrent infection after internal fixation. Therefore, we took re-operation to remove the internal fixator for infection control, patients recovered after conservative treatment such as immobilization and systemic anti infection. The numerical value of leukocytes, C-reactive protein, ESR and VAS scores of the two groups were significantly lower than those before surgery, the difference was statistically significant $(\mathrm{P}<0.001)$. In Group NVO, 16 cases recovered from Asia grade D to grade E, 1 case from grade C to grade D; 10 cases in Group PVO recovered from grade D to grade $\mathrm{E}$ and 2 cases recovered from grade $\mathrm{C}$ to grade $\mathrm{D}$. There was no significant difference between these two groups $(\mathrm{P}>0.05)$. By univariate analysis, multiple vertebral involvement and abscess formation $(\mathrm{P}=0.003, \mathrm{P}=$ 0.025) were significantly associated with PS recurrence; there was a tendency for PS recurrence among microbial infection $(\mathrm{OR}=1.889)$, spinal prosthesis $(\mathrm{OR}=7.083)$ and allogenic bone ( $\mathrm{OR}=2.032$ ), yet not obvious. By multivariate analysis, we found that multiple 
vertebral involvement $(\mathrm{OR}=12.656,95 \% \mathrm{CI}: 1.536-104.303, \mathrm{P}=0.018)$ was a risk factor for PS recurrence.

\section{Conclusion}

The treatment of PVO is more challenging than NVO, especially in the cases of spinal implant infection. Although the antibiotic treatment time of PVO is longer than that of NVO, the recurrence rate of PVO is higher. Longer antibiotic therapy and, if necessary, surgical debridement or removal of implants are important approaches to successful treatment of PVO.

\section{Key Words}

Vertebral Osteomyelitis, Spinal Surgery, Suppuration, Outcome

\section{Introductions}

Pyogenic spondylodiscitis (PS) is a non-specific, acute or subacute inflammatory disease of the spine infected by pyogenic bacteria, which involves the vertebral bodies, intervertebral discs and surrounding soft tissues. PS not only destroys the normal structure and stability of the local spine, may also affect the neurological function and can lead to bacteremia and sepsis. The mortality rate of PS has been reported between $4 \%-29 \%$.

PS predominantly occurs in elderly aged between 50-70 years and the chronic debilitated patients [1]. The proportion of PS in males and females is 1.6-2.0:1. PS is relatively rare, it is reported that the incidence rate is between 0.2-2 per 100 thousand people every year [2]. However, recent studies have reported alarming increases in incidence over the past 20 years. Denmark conducted a nationwide study over the past 14 years, which showed that the annual incidence rate of PS increased from 2.2 to 5.8 / 100000 [3]. Akiyama[4]et al. reported an increase of incidence of approximately $140 \%$ in Japan. PS can be caused by a variety of microorganisms, including Staphylococcus aureus and Escherichia coli [5] as we saw common, and others for instance, Streptococcus, pneumococcus, Salmonella, Pseudomonas and Candida. Nearly $9 \%$ of vertebral osteomyelitis is caused by polymicrobial infections.

Despite a significant downward trend in PS mortality, it still has a high incidence rate, which places a significant burden on patients and the medical system.A large number of studies have shown that the treatment of PS is still lack of unified treatment plan and standard, including the best duration of antibiotic treatment has not been clearly defined; on the other hand there is no uniform standard for surgical indications and surgical treatment. 
The two common types of PS are postoperative vertebral osteomyelitis (PVO) and native vertebral osteomyelitis (NVO). Although both types are caused by purulent bacteria, but the pathogenesis, clinical manifestations, treatment methods, treatment time, prognosis, complications and recurrence rate of the two spinal infections are different. Many scholars have begun to pay attention to the treatment and efficacy of PS in recent years, and related literature has also been reported, however there are few reports comparing PVO and NVO. The purpose of this study is to compare and describe the microbiology, clinical characteristics, treatment and efficacy of $\mathrm{PVO}$ and NVO, and analyze the related factors from pathogenesis to prognosis, so as to provide scientific basis for making standard diagnosis and treatment plan of PVO and NVO respectively.

\section{Materials and Methods}

\section{General information}

The clinical data of patients with PS admitted to Spine Surgery Department, the First Affiliated Hospital of Xinjiang Medical University from January 2010 to December 2019 had been retrospectively analyzed. Including general information of patients, comorbidity, symptoms, microbial culture, X-ray, MRI, CT features, laboratory data(including leukocyte count, ESR and CRP), VAS score, antibiotic treatment and postoperative curative effect, neurological improvement and recurrence rate. The diagnostic criteria of PS were as follows[6][7]: (1) Clinical symptoms of suspected PS (pain on the back and pain after resting could not be relieved after moving, neurological symptoms could be found, etc.); (2) MRI features were consistent with PS (endplate destruction, intervertebral disc inflammation, intervertebral space, paravertebral soft tissue and epidural necrosis or abscess); (3) Main inflammatory indicators: increased $\mathrm{ESR}(>20 \mathrm{~mm} / \mathrm{h}), \mathrm{CRP}(>10 \mathrm{mg} / \mathrm{L})$, fever $\left(\mathrm{T}>38{ }^{\circ} \mathrm{C}\right)$;

(4) Positive blood culture, twice; (5) Pathogens isolated from spine or paravertebral space. If (5) is added to any one of the first three items, PS can be diagnosed; if (4) is added to any one of the first three items, PS is highly suspected; if any two of the first three items are added, PS may be considered. PVO was defined as patients who had residual implants at the time of diagnosis of vertebral osteomyelitis (VO) or who underwent spinal surgery at the same location as the infection within one year after the diagnosis of VO. According to the above diagnostic criteria, 52 patients were finally included, including 30 cases of NVO and 22 cases of PVO. 
Among the 52 patients, there were 30 patients in Group NVO, including 18 males and 12 females aged from 15 to 73 years old, with an average age of (50.47 \pm 20.45 ), and 22 patients in Group PVO, including 13 males and 9 females aged from 14 to 73 years old, with an average age of (51.45 \pm 16.97$)$. All patients presented with varying degrees of local pain, 21 patients (12 cases in Group NVO and 9 cases in Group PVO) had fever symptoms, 19 patients (12 cases in Group NVO and 7 cases in Group PVO) had limb pain and numbness; the visual analogue scale score (VAS) was $3-9$ pts, with an average of $(7.10 \pm 1.30)$ in Group NVO and $(5.73 \pm$ 0.985) in Group PVO. There were 15 patients with diabetes mellitus (7 patients in Group NVO and 8 patients in Group PVO), 10 patients with hypertension (6 patients in Group NVO and 4 patients in Group PVO). 5 patients in Group NVO had concurrent other infections. Among them, 2 patients had a history of urinary tract infection within 1 month before the onset of low back pain symptoms, 2 patients had a history of respiratory infection within 1 month before visiting the hospital, and 1 patient had a history of postoperative infection of knee joint. Multiple vertebral bodies were involved in 9 patients (2 in Group NVO and 7 in Group PVO). In Group NVO, there were 11 cases of epidural abscess and 10 cases of paraspinal abscess; 9 cases of epidural abscess and 9 cases of paraspinal abscess in Group PVO. 29 patients had neurological dysfunction before surgery. According to the American Spinal Injury Association (Asia) neurological function classification, 26 cases were classified as grade D (16 cases in Group NVO, 10 cases in Group PVO), and 3 cases in Grade C(1 case in Group NVO and 2 cases in Group PVO). Leukocyte count was normal or slightly higher than normal after admission, $(8.35 \pm 2.88) \times 109 / \mathrm{L}$ in Group NVO and $(9.80 \pm 3.53) \times 109 / \mathrm{L}$ in Group PVO, and the dynamic erythrocyte sedimentation rate (ESR) in Group NVO was $(62.07 \pm 16.94) \mathrm{mm} / 1 \mathrm{~h}$ and that in Group PVO was $(58.86 \pm 15.51) \mathrm{mm} / 1 \mathrm{~h}$. The level of CRP increased $(35.38 \pm 24.76) \mathrm{mg} / \mathrm{L}$ in Group NVO and (83.49 \pm 58.10$) \mathrm{mg} / \mathrm{L}$ in Group PVO ( Table 1 ) .

The lesions were located in different parts of the whole spine, in Group NVO there were 23 cases (76.7\%) found on lumbar, 5 cases (16.7\%) found on thoracic, 2 cases $(6.7 \%)$ found on cervical; 16 cases $(72.7 \%)$ found on lumbar vertebrae and 6 cases $(27.3 \%)$ found on thoracic vertebrae in Group PVO. Typical radiographic findings of patients were varying degrees of intervertebral space narrowing and bone destruction. MRI exams showed that hyperintense 
signal on T2WI of diseased vertebral body and intervertebral space with disappearance of fissure-like hypointense in diseased disc nucleus pulposus, and T1WI manifested as hypointense with loss of vertebral body and endplate contour. 3-D reconstruction CT scan could show the destruction of vertebral body more clearly, which is helpful for the selection of preoperative surgical scheme. (Table 1)

The study was approved by the ethical committee of the First Affiliated Hospital of Xinjiang Medical University, China. The procedures followed were in accordance with the ethical standards of Helsinki Declaration. All experiments were performed in accordance with relevant guidelines and regulations. Informed consent was obtained from all subjects or, if subjects are under 18 , from a parent and/or legal guardian.

\section{Preoperative management}

Bacterial culture and drug sensitivity tests were performed before antibiotics were administered to all patients after admission. Blood culture and drug sensitivity tests were performed for patients with body temperature over $38^{\circ} \mathrm{C}$. Patients with positive bacterial culture were treated with sensitive antibiotics according to drug sensitivity test; patients with negative bacterial culture were treated with empirical broad-spectrum antibiotics and monitored more closely. The total course of intravenous antibiotics was more than 6 weeks.

Surgical treatment is required when neurological dysfunction, conservative treatment failure, spinal instability (spinal instability includes segmental kyphosis $>15^{\circ}$, vertebral collapse $>50 \%$, and displacement $>5 \mathrm{~mm}$.), huge paravertebral abscess or epidural abscess, and severe pain occur[8]. In Group NVO, 2 cases were treated with antibiotics only, the other 2 cases were treated with posterior debridement and catheter drainage, 23 cases (6 cases in anterior approach and 17 cases in posterior approach) were treated with debridement, fusion and internal fixation, and 3 cases ( 2 cases in anterior approach and 1 case in posterior approach) were treated with debridement and bone graft fusion; in Group PVO, 13 patients underwent posterior debridement and catheter drainage, 1 patient underwent posterior debridement, fusion and internal fixation, and 3 patients (1 anterior and 2 posterior) 
underwent debridement and bone graft fusion; ( Tab 2 ) in Group NVO, 1 patient had recurrent infection, so lesion removal and implant removal were performed; 5 patients in Group PVO underwent lesion removal and implant removal (Table 2).The removed lesion tissues were sent for pathological examination, bacterial culture and drug sensitivity tests.

\section{Follow-up evaluation and statistical analysis}

All patients were followed up at 1,3,6 and 12 months after operation, including clinical symptoms, leucocyte count, ESR and CRP to evaluate infection control, VAS score to evaluate pain improvement, Asia neurological function classification to evaluate neurological function improvement,etc.; at the same time, X-ray and three-dimensional CT scanning were performed to evaluate the lesion repair, bone graft fusion and internal fixation, and MRI scanning was performed if necessary to detect the healing of the diseased vertebrae and the condition of the spinal cord.

All statistical analyses were performed with SPSS 26.0 statistical software. The measurement data accorded with normal distribution or approximate normal distribution( $\overline{\mathrm{x}}$ $\pm \mathrm{S}$ ), the differences between the two groups were compared by t-test, the partial distribution data was expressed by $\mathrm{M}(\mathrm{P} 25, \mathrm{p} 75)$, the differences between the two groups were compared by Mann Whitney U test, the value of count data cases was expressed as(\%), the differences between the two groups were compared by C2-test or Fisher exact test. Univariate and multivariate logistic regression analyses were used to identify risk factors associated with treatment failure or relapse. Variables with $\mathrm{P}<0.10$ after univariate analysis were included in multivariate analysis. Significance tests were two-tailed, and P-values $<0.05$ indicated statistical significance.

\section{Results}

60\% patients in Group NVO and 59.1\% in Group PVO were males. The proportion of male patients in both groups was higher than that of female patients, but there was no significant difference between the two groups in gender $(\mathrm{P}>0.05)$. Diabetes was the most 
common comorbidity (Group NVO 23.3\%, Group PVO 36.4\%), more common in Group PVO, but the difference was not statistically significant $(\mathrm{P}=0.306)$ (Table.1); followed by hypertension (Group NVO 20\%, Group PVO 18.2\%), there was no significant difference between the two groups $(\mathrm{P}>0.05)$.Synchronous infection $(<30$ days $)$ was more common in Group NVO (16.7\% vs. 0\%, P < 0.001). The most common symptom was local pain(52 / 52, accounting for 100\%). Other symptoms included fever (40\% in Group NVO and $40.1 \%$ in PVO group), limb pain and numbness (40\% in Group NVO , 31.8\% in Group PVO), and neurological dysfunction ( $56.7 \%$ in Group NVO , $54.5 \%$ in Group PVO). There was no significant difference between these two groups ( $\mathrm{P}=0.947, \mathrm{P}=0.545, \mathrm{P}=1.000)$. Histopathological examination showed that fibrous tissue was proliferative and degenerative, with infiltration of plasma cells, lymphocytes, neutrophils and other acute and chronic inflammatory cells, and thin yellow pus with necrosis could be found.

Multiple vertebral involvement was more common in Group PVO, and the difference was statistically significant $(\mathrm{P}=\mathrm{0.046})$. The proportion of patients with epidural abscess and paraspinal abscess formation was not significantly different between the two groups $(\mathrm{P}>$ 0.05).

Table 3 shows the pathogenic bacteria in NVO and PVO groups. Among 52 patients, 35 cases were positive for bacterial culture (16 cases in Group NVO and 19 cases in Group PVO). The positive rate was 53.3\% in Group NVO and $86.4 \%$ in Group PVO. Staphylococcus aureus was the most common microorganism (11 / 52 cases, 21.2\%). There were 6 cases (20\%) in Group NVO and 5 cases (22.7\%) in Group PVO, there was no significant difference between the two groups.

The time of intravenous antibiotic treatment in NVO group and PVO group was (38.40 \pm 12.64) days and $(37.50 \pm 9.49)$ days, and the oral antibiotic treatment time was $(24.33 \pm 9.10)$ days and (33.44 \pm 17.14$)$ days, respectively. The total antibiotic treatment time was $(62.73 \pm$ 15.28) days and $70.94 \pm 15.90$ days, respectively. The total time of antibiotic treatment and oral antibiotic treatment in PVO group was longer than that in NVO group, but the difference was not statistically significant $(\mathrm{P}=0.094, \mathrm{P}=0.062)$. Compared with $\mathrm{PVO}$ group, NVO 
group had more common treatment methods of debridement, fusion and internal fixation, and the difference was statistically significant $(\mathrm{P}<0.001)$. However, $\mathrm{PVO}$ group had more frequent debridement and catheter drainage than NVO group, and the difference was statistically significant $(\mathrm{P}<0.001)$.

All patients were followed up for 12-24 months. Up to the last follow-up, 3 patients in NVO group had recurrence, the recurrence rate was 10\% (3 / 30), 9 patients in PVO group had recurrence, the recurrence rate was $40.1 \%(9 / 22)$, the recurrence rate of PVO group was higher, the difference was statistically significant $(\mathrm{P}=$ 0.009). 6 months after operation, 8 cases (26.7\%) in NVO group and 6 cases (27.3\%) in PVO group had residual back pain; 4 cases (13.3\%) in NVO group and 5 cases (22.7\%) in PVO group had residual lower limb pain and discomfort, and the patients recovered after conservative treatment such as immobilization and physiotherapy. In 44 patients with spinal internal fixation, 13.6\% (1 NVO, 5 PVO) had recurrent infection after internal fixation, so we decided to take out the internal fixation device to control the infection, and the patient recovered after conservative treatment such as immobilization and systemic anti infection.(fig 1)

The scores of white blood cell, C-reactive protein, ESR and VAS in the two groups were significantly lower than those before operation at 1, 3, 6 months and the last follow-up, and the differences were statistically significant $(\mathrm{P}<\mathrm{0.001})$ (Table 4) The neurological function of the patients improved significantly after operation. In NVO group, 16 patients' ASIA grade was restored from D to E, 1 from $\mathrm{C}$ to $\mathrm{D}$; in PVO group ,10 from D to $\mathrm{E}$ and 2 from $\mathrm{C}$ to $\mathrm{D}$. There was no significant difference between the two groups ( $\mathrm{P}>0.05)$ (Tab.4).(fig 2)

Table 5 shows the risk factors of PS recurrence. In univariate analysis, multiple vertebral body involvement and abscess formation were significantly correlated with PS recurrence (P = 0.003, $\mathrm{P}=0.025)$; microbial infection $(\mathrm{OR}=1.889)$, spinal prosthesis $(\mathrm{OR}=7.083)$ and allogeneic bone $(\mathrm{OR}=1.2 .032)$ had a trend, but not very obvious. In multivariate analysis, we found that multiple vertebral involvement (OR: 12.656, 95\% CI: 1.536-104.303, P = 0.018) was a risk factor for PS recurrence. 


\section{Discussion}

PS refers to the infection of vertebral body, intervertebral disc and soft tissue around vertebral body caused by pyogenic bacteria [9], it takes up $4 \%$ of all kinds of osteomyelitis, including vertebral osteomyelitis, discitis and epidural abscess [10]. Improper or delayed treatment of PS may lead to serious complications. Its incidence is on the rise, and although there has been great progress in the understanding of this disease, doctors are still facing great challenges about the diagnosis and treatment [11]. Despite surgery and antibiotic treatment, the clinical outcomes yet remain poor [12].

In our study, as a part of non-specific clinical manifestations, pain was the most common symptom. All patients showed local pain (52 / 52, accounting for 100\%), which was similar to the results of Hopkinson $\mathrm{N}$ et al. [13].In Group NVO, the local symptoms of low back pain were more severe than the patients in Group PVO, the VAS score of Group NVO was (7.10 \pm 1.30), which was higher than that of Group PVO ( $5.73 \pm 0.985)$ before surgery.

Most studies have shown that white blood cells and neutrophils are not the sensitive factors for diagnosing PS. Yoon et al. [14] pointed out that only $42.6 \%-81.3 \%$ patients with suppurative spondylitis had elevated leucocyte count. Domestic studies such as Polat [15] and others showed that $58 \%$ patients with PS had elevated neutrophil and leucocyte count. On the contrary, erythrocyte sedimentation rate(ESR) and C-reactive protein(CRP) were more sensitive, the sensitivity was $98 \%$ and $100 \%$ respectively, but the specificity of ESR and CRP was poor, which can be used to observe the progress of disease and evaluate the curative effect [16]. Like the results reported by Yoon and Polat et al., 26.7\% patients in Group NVO and 40.1\% patients in Group PVO increased the leucocyte count in this study. Although the leucocyte count in Group PVO increased more significantly, the difference was not statistically significant(P > 0.05), and all patients showed increasing ESR and CRP, so we think that ESR and CRP detection is more sensitive than leucocyte count. Although the specificity of CRP is low, Jean et al. [17] found that CRP value over $63 \mathrm{mg} / \mathrm{L}$ was signifcantly associated with shorter diagnostic delay. Therefore, the association of spinal pain and high value of CRP could increase the suspicion of PS and lead to early diagnosis.

Identification of pathogens is the cornerstone of PS diagnosis and treatment. CT-guided biopsy is often needed for patients without surgical indications. The specificity of CT-guided biopsy was $99 \%$, and yet the sensitivity was $52 \%$ - $91 \%$ [18]. Spira et al. [19] reported that the positive rate of CT-guided biopsy in 34 patients with PS was 82.3\%. Marschall et al. [20] showed that the positive rate of open biopsy culture in PS patients was 91\%, while that of percutaneous biopsy was only 53\%.The common gram-positive pathogens include Staphylococcus aureus, Staphylococcus epidermidis, Streptococcus and Enterococcus, among which Staphylococcus aureus is the most common; while Gram-negative bacteria include Escherichia coli, Pseudomonas aeruginosa and Proteus mirabilis [21] .This study showed that the positive rate of bacterial culture was 53.3\% in Group NVO and $86.4 \%$ in Group PVO. The positive rate of Group PVO was higher than that of Group NVO. The results were similar to those reported in the literature, and basically a common pathogenic bacterial infection. Besides, due to PS is mainly blood-borne infection, the cause of bacteremia is often difficult to identify in clinical. In this study, among the patients with positive etiology in Group NVO, 2 patients had a clear history of urinary tract infection before pain occured in the lower back or thoracodorsal region, and the etiology of urinary system was consistent with blood culture; 2 
patients complained of previous respiratory infections, 1 patient had postoperative infection history of knee joint, and other patients denied having other infectious history before treatment. Therefore, we believe that respiratory and urinary infections are an indispensable source of infection for PS.

As for the duration of antibiotic treatment for PS, most literatures suggest that 6-12 weeks should be the standard of treatment. IDSA (Infectious Diseases Society of America) clinical guidelines believe that most patients with PS need 6 weeks of antibiotic treatment. Currently one certain study shows that there is no significant difference between 6 weeks and 12 weeks of antibiotic treatment for PS patients [22]. But this study excluded patients with negative microbial cultures, which could affect the results. Roblot et al.[23] compared the risk of infection recurrence between patients with anti infection course $\leq 6$ weeks and $\geq 6$ weeks, and there was no significant difference in the risk of infection recurrence. Grados et al.[24] suggested that the total duration of antibiotic treatment for 12 weeks could reduce the risk of recurrence, and the recurrence rate of 8-week antibiotic use in other studies decreased from $10 \%$ to $3.9 \%$. Li et al.'s[25] latest research shows that oral antibiotics are no worse than intravenous antibiotics in the treatment of bone and joint infections if the treatment is started within 6 weeks after the infection.In this study, the total time of antibiotic treatment in Group NVO and Group PVO was $(62.73 \pm 15.28)$ days and $70.94 \pm 15.90$ days respectively, which achieved satisfactory results. Although the total time of antibiotic treatment in Group PVO was longer than that in Group NVO, the difference was not statistically significant $(\mathrm{P}=0.094)$. In our patients with recurrence related risk factors (such as abscess in the surrounding tissue of the spine, multiple vertebral body involvement, etc.), the antibiotic treatment time was longer ( $>8$ weeks), which was similar to the results of Park et al.[26].

Systemic support therapy, antibiotic therapy and local immobilization are the preferred choices for PS. But surgical treatment is still recommended for patients with neurological impairment, conservative treatment failure, spinal instability, huge paravertebral abscess or epidural abscess, and severe pain. Spinal instability includes segmental kyphosis $>15^{\circ}$, vertebral collapse $>50 \%$, and displacement $>5 \mathrm{~mm}[8]$.

At present, the choice of surgical approach for PS is still controversial. The anterior approach can make the infected part fully exposed, can directly and clearly see the lesion tissue, and then can fully and thoroughly remove the necrotic and infected tissue. At the same time, it can fully drain the concurrent abscess, so as to prevent the residual infection focus and bacterial planting. However, because the anterior internal fixation is not strong enough and the risk of infection is high, it is easy to lead to surgical failure. There are also some scholars through the posterior approach debridement and get better results, nerve root decompression, deformity correction is suitable for posterior surgery. According to this point of view, the combination of anterior and posterior surgery is the best choice for PS surgery. However, due to the large trauma and long operation time, especially for the elderly patients, it is not often used, which is still controversial. Since the anterior approach is associated with higher incidence rate and mortality, recent literature has shown that the posterior approach can adequately solve the infection problem [27] .In this study, 17 patients in Group NVO and 1 patient in Group PVO were treated with posterior debridement, fusion and internal fixation; 6 patients in Group NVO were treated with anterior debridement, fusion and internal fixation; 2 patients in Group NVO and 13 patients in Group PVO were treated with simple debridement 
and catheter drainage, and all patients achieved satisfactory results. This is like the research results of Polat [15] et al and Tang [28] at al.. Operative methods of PVO and NVO in PS are different, so we think that PVO is usually the original incision approach, and NVO is based on the location, scope and technical conditions of the lesions to choose the appropriate surgical method for patients.

Structural bone grafting is often performed after thorough debridement, although there are some controversies in the past about the introduction of allogenic bone into the field of surgery, yet some other studies have found that autogenous bone and allogeneic bone have similar recurrence rate and clinical outcomes [29] . In our study, 14 patients in Group NVO used autologous bone, 6 patients used allogeneic bone; as for Group PVO, 1 patient used autologous bone, 3 patients used allogeneic bone; within the two groups, autologous bone and allogeneic bone had similar clinical outcomes, consistent with previous research reports. However, we believe that fusion in the case of infection is challenging and we suggest that autologous bone graft should be used as far as possible.

The latest development of minimally invasive surgery(MIS), such as percutaneous endoscopic debridement combined with percutaneous pedicle screw fixation provides an alternative method for the treatment of PS [30]. Matsubara et al. [31] found that percutaneous aspiration and drainage (PSAD) could be used for pathogen identification, histopathological diagnosis and even simultaneous treatment. Omran and Ibrahim et al. [32] performed posterior debridement, decompression and interbody fusion in 25 patients with lumbar suppurative discitis, postoperative satisfactory results were obtained in terms of fusion rate, pain and neurological improvement. Minimally invasive techniques may be the developing trend for PS surgery.

This study found that treatment for PVO may be more challenging than which for NVO, especially the cases with spinal implant infection, in this situation successful treatment required longer antibiotic therapy or implant removal. Although the duration of antibiotic treatment in Group PVO was longer than that in Group NVO, the difference was not statistically significant $(\mathrm{P}=0.094)$. Just as the results reported by Kim et al. [33], 13.6\%(1 NVO and 5 PVO) patients had recurrent infection after internal fixation, so we decided to take out the internal fixation device to control the infection, and the patients recovered after conservative treatment such as immobilization and systemic anti infection. Recently, there are more reports about PVO treatment failure rate, from $16 \%$ to $29 \%$ [34,35]. In this study, 3 patients from Group NVO relapsed with a recurrence rate of 10\%(3 / 30), and 9 patients from Group PVO relapsed with a recurrence rate of 40.1\% (9/22). The recurrence rate of Group PVO was higher than that of Group PVO, and the difference was statistically significant $(\mathrm{P}=$ 0.009). In addition, in terms of residual disease and recurrence, NVO group was significantly better than Group PVO. Although there are no standard guidelines for the treatment of PVO, longer antibiotic therapy and surgical debridement or implant removal if necessary are important for successful treatment of PVO.

Our study had some limitations. First, for it belongs to a single-center retrospective study, some patients were lost to follow-up. The sample size is not large enough, the conclusion still needs to expand the sample size for further research verification. Further studies especially clinical trials,are needed, such as the role of FDG-PET / CT in the diagnosis of PS, the practical value of MRI in follow-ups, and the optimal duration of antibiotic treatment in 
culture-negative PS, etc.

\section{Conclusion}

PS can be triggered by a variety of microorganisms especially staphylococcus aureus. It is difficult to diagnose, but with CT-guided needle biopsy and bacterial culture we may do the diagnosis more effectively. PVO is one of the challenges for doctors may face during clinical work. We should choose different surgical plan according to the patient's condition, infection site, infection range, spinal stability on the basis of anti infection treatment.

\section{Availability of data and materials}

The datasets used and analyzed during the current study are available from the corresponding author on reasonable request.

\section{Abbreviations}

VAS:visual analogue scale

CT:computed tomography

MRI:magnetic resonance imaging

ESR:rythrocyte sedimentation rate

CRP:C-reactive protein

PVO:Postoperative Vertebral Osteomyelitis

NVO:native vertebral osteomyelitis .

\section{References}

1. Yong yuan Zhang, Honghui Sun, Dingjun Hao, et al . Progress in the diagnosis and treatment of suppurative spinal infection[J]. Chinese Journal of Spine and Spinal Cord,2019,29(8):747-751.

2.Cheung W Y, Luk K D. Pyogenic spondylitis[J]. Int Orthop,2012,36(2):397-404. PMID: 22033610

3. Kehrer M, Pedersen C, Jensen T G, et al. Increasing incidence of pyogenic spondylodiscitis: a 14-year population-based study[J]. J Infect,2014,68(4):313-320. 
4. Akiyama T, Chikuda $\mathrm{H}$, Yasunaga $\mathrm{H}$, et al. Incidence and risk factors for mortality of vertebral osteomyelitis: a retrospective analysis using the Japanese diagnosis procedure combination database[J]. Bmj Open,2013,3(3):e2412.

5. Rutges J P, Kempen D H, van Dijk M, et al. Outcome of conservative and surgical treatment of pyogenic spondylodiscitis: a systematic literature review[J]. Eur Spine J,2016,25(4):983-999.

6. KangS J, Jang H C, Jung S I, et al. Clinical characteristics and risk factors of pyogenic spondylitis caused by gram-negative bacteria[J]. PLoS One,2015,10(5):e127126.

7. Viezens L, Schaefer C, Helmers R, et al. Spontaneous Pyogenic Spondylodiscitis in the Thoracic or Lumbar Spine: A Retrospective Cohort Study Comparing the Safety and Efficacy of Minimally Invasive and Open Surgery Over a Nine-Year Period[J]. World Neurosurg,2017,102:18-27.

8. Aboobakar R, Cheddie S, Singh B. Surgical management of psoas abscess in the Human Immunodeficiency Virus era[J]. Asian J Surg,2018,41(2):131-135.

9. Kourbeti I S, Tsiodras S, Boumpas D T. Spinal infections: evolving concepts[J]. Curr Opin Rheumatol,2008,20(4):471-479.

10. Bornemann R, Muller-Broich J D, Deml M, et al. [Diagnosis and Treatment of Spondylodiscitis/Spondylitis in Clinical Practice][J]. Z Orthop Unfall,2015,153(5):540-545.

11. Kehrer M, Pedersen C, Jensen T G, et al. Increasing incidence of pyogenic spondylodiscitis: a 14-year population-based study[J]. J Infect,2014,68(4):313-320.

12. Lemaignen A, Ghout I, Dinh A, et al. Characteristics of and risk factors for severe neurological deficit in patients with pyogenic vertebral osteomyelitis: A case-control study[J]. Medicine (Baltimore),2017,96(21):e6387.

13. Hopkinson N, Patel K. Clinical features of septic discitis in the UK: a retrospective case ascertainment study and review of management recommendations[J]. Rheumatol Int,2016,36(9):1319-1326. 
14. Kwon, H. H, Yoon, et al. Differential diagnosis between tuberculous spondylodiscitis and pyogenic spontaneous spondylodiscitis: a multicenter descriptive and comparative study[J]. The spine journal: official journal of the North American Spine Society,2015.

15. Polat Mamat, Weibin Sheng, Li Cao, et al. Diagnosis and surgical treatment of suppurative spinal infection[J]. Chinese Journal of Bone and Joint Injury,2008,023(9):756-757.

16. Yongxian Li , Shuncong Zhang, Ling Mo , et al. (Research )progress in diagnosis and treatment of pyogenic spondylitis[J]. Journal of Spine Surgery,2016,14(5):316-320.

17. Jean M, Irisson J O, Gras G, et al. Diagnostic delay of pyogenic vertebral osteomyelitis and its associated factors[J]. Scand $J$ Rheumatol,2017,46(1):64-68.

18. Pupaibool J, Vasoo S, Erwin P J, et al. The utility of image-guided percutaneous needle aspiration biopsy for the diagnosis of spontaneous vertebral osteomyelitis: a systematic review and meta-analysis[J]. Spine J,2015,15(1):122-131.

19. Spira D, Germann T, Lehner B, et al. CT-Guided Biopsy in Suspected Spondylodiscitis--The Association of Paravertebral Inflammation with Microbial Pathogen Detection[J]. PLoS One,2016,11(1):e146399.

20. Marschall J, Bhavan K P, Olsen M A, et al. The impact of prebiopsy antibiotics on pathogen recovery in hematogenous vertebral osteomyelitis[J]. Clin Infect Dis,2011,52(7):867-872.

21. Nickerson EK, Sinha R. Vertebral osteomyelitis in adults: an update. Br Med Bull. 2016;117(1):121-138.

22. Bernard L, Dinh A, Ghout I, et al. Antibiotic treatment for 6 weeks versus 12 weeks in patients with pyogenic vertebral osteomyelitis: an open-label, non-inferiority, randomised, controlled trial[J]. Lancet,2015,385(9971):875-882. 
23. Roblot F, Besnier J M, Juhel L, et al. Optimal Duration of Antibiotic Therapy in Vertebral Osteomyelitis[J]. Seminars in Arthritis \& Rheumatism,2007,36(5):269-277.

24. Grados F, Lescure F X, Senneville E, et al. Suggestions for managing pyogenic (non-tuberculous) discitis in adults[J]. Joint Bone Spine,2007,74(2):133-139.

25. Li H K, Rombach I, Zambellas R, et al. Oral versus Intravenous Antibiotics for Bone and Joint Infection[J]. N Engl J Med,2019,380(5):425-436.

26. Park K H, Cho O H, Lee J H, et al. Optimal Duration of Antibiotic Therapy in Patients With Hematogenous Vertebral Osteomyelitis at Low Risk and High Risk of Recurrence[J]. Clin Infect Dis,2016,62(10):1262-1269.

27. Ahmed Aljawadia, Noman Jahangira, Ana Jeelania, Zak Fergusona, Noman Niazia, Frances Arnallb, Anand Pillai, et al. Management of Pyogenic Spinal Infection, review of literature.[J]. Journal of Orthopadics 16(2019) 508-512. doi.org/10.1016/j.jor.2019.08.014.

28. Kai Tang, Shibing Qin , Weijie Dong, Tinglong Lan, Jun Fan, GuangxuanYan , Heng Wang , et al. Observation of the efficacy of one-stage posterior debridement and bone grafting and internal fixation in the treatment of suppurative spondylitis[C]. Journal of Chinese Journal of Tuberculosis , Beijing Chest Hospital Affiliated to Capital Medical University, Bone and Joint Professional Committee of the Tuberculosis Clinical Professional Branch of China National Defense Tuberculosis Association. The 6th Bone and Joint Tuberculosis Clinical Diagnosis and Treatment Progress and Standardization Symposium. Journal of Chinese Journal of National Defence Tuberculosis Society, Beijing Chest Hospital Affiliated to Capital Medical University, Bone and Joint Professional Committee of Tuberculosis Clinical Branch of China National Tuberculosis Association: China National Tuberculosis Association, 2018: 45-50.

29. Kim HW, Ryu J-I, Bak KH. The safety and efficacy of cadaveric allografts and titanium cage as a fusion substitutes in pyogenic osteomyelitis. $J$ Korean Neurosurg Soc.2011;50(4):348-56.

30. Kaidi Duan, Yi Qin Jichao Ye, Wei Zhang, Xumin Hu, Jinlang Zhou, Liangbin Gao, Yong Tang, et al . Percutaneous endoscopic debridement with percutaneous pedicle screw fixation for lumbar pyogenic spondylodiscitis: a preliminary study[J]. Int Orthop ,2019,Dec,26. doi: 10.1007/s00264-019-04456-1. [Epub ahead of print]. 
31. Matsubara T ,Yamada K,Sato K , Gotoh M ,Nagata K, et al .Clinical outcomes of percutaneous suction aspiration and drainage for the treatment of infective spondylodiscitis with paravertebral and/or epidural absess .Spine J.18(9):1558-1669. doi:10.1016/j.spinee.2018.02.020.

32. Omran K,Ibrahim AH,et al. Outcome of transforminal lumber thorough debridement ,decompression ,and spondylodesis technique in treatment of 25 patients with pyogenic spondylodiscitis [J]. World Neurosurg .2019.[Epub ahead of print].

33. Kim UJ, Bae JY, Kim SE, et al. Comparison of pyogenic postoperative and native vertebral osteomyelitis. Spine J. 2019;19(5):880-887. doi:10.1016/j.spinee.2018.11.012

34. Wille H, Dauchy FA, Desclaux A, Dutronc H, Vareil MO, Dubois V, et al. Efficacy of debridement, antibiotic therapy and implant retention within three months during postoperative instrumented spine infections. Infect Dis (Lond). 2017;49:261-7.

35. Adam D, Papacocea T, Hornea I, Croitoru R. Postoperative spondylodiscitis. A review of 24 consecutive patients. Chirurgia (Bucur). 2014;109:90-4.

\section{Acknowledgements}

Not applicable.

\section{Funding}

This study did not receive any funding.

\section{Author information}

\section{Affiliations}

Maimaitiaili Abudurexiti ${ }^{1}$, Mulade Maierdan ${ }^{2}$, Tao Xu ${ }^{3}$, Xieraili Saimaiti ${ }^{4}$, Wei-Bing Sheng 5 , Yang Zhou ${ }^{6}$, Maierdan Maimaiti ${ }^{1}$ 
1, 3, 4, 5,6 Department of spine surgery, First Affiliated Hospital of Xinjiang Medical University, Urumqi 830054, Xinjiang Uyghur Autonomous Region, China.

${ }^{2}$ Grade 2018 in clinical Medicine of Tongji University Medical School, Shanghai 200092, China.

\section{Contributions}

M A : project conceptualization, data collection/validation, result interpretation, reporting \& editing, and final approval of the version to be submitted.

M M , T X , X S , Y Z : project conceptualization, result interpretation .

W B S : final approval of the version to be submitted, and project guarantor.

All authors have read and approved this manuscript.

\section{Corresponding Author}

Maierdan Maimaiti , MD, Department of spine surgery, First Affiliated Hospital of Xinjiang Medical University, Urumqi 830054, Xinjiang Uyghur Autonomous Region, China; E-mail: mardanmmtmx@163.com, Tel: +86 o9914365316, Fax: +8609914365316.

\section{Ethics declarations}

\section{Ethics approval and consent to participate}

The study protocol was approved by First Affiliated Hospital of Xinjiang Medical University . All study participants provided written informed consent before being enrolled in this study.

\section{Consent for publication}

Consent for publication obtained from participants.

\section{Competing interests}


The authors declare that they have no competing interests .

\section{Availability of Data and Materials statement}

The datasets generated during and analysed during the current study are not publicly available due to Data and Materials were used under license for the current study, and so are not publicly available, but are available from the corresponding author on reasonable request"

\section{Additional information}

\section{Publisher's Note}

\section{N/A}

\section{Rights and permissions}

Open Access This article is licensed under a Creative Commons Attribution 4.0 International License, which permits use, sharing, adaptation, distribution and reproduction in any medium or format, as long as you give appropriate credit to the original author(s) and the source, provide a link to the Creative Commons licence, and indicate if changes were made. The images or other third party material in this article are included in the article's Creative Commons licence, unless indicated otherwise in a credit line to the material. If material is not included in the article's Creative Commons licence and your intended use is not permitted by statutory regulation or exceeds the permitted use, you will need to obtain permission directly from the copyright holder. To view a copy of this licence, visit http://creativecommons.org/licenses/by/4.0/. The Creative Commons Public Domain Dedication waiver (http://creativecommons.org/publicdomain/zero/1.o/) applies to the data made available in this article, unless otherwise stated in a credit line to the data. 


\section{Figure legends}

Fig. 1 Male, 62 years old, (a b c d) Show the preoperative anteroposterior and lateral, flexion and hyperextension radiographs. The results showed that the physiological curvature of lumbar spine decreased, lumbar bone hyperplasia, L2-3, L4-5, L5-S1 intervertebral space narrowed, L4-5 vertebral instability. (e f) Show preoperative MRI of the patient, showing lumbar degenerative disease, L2-3, L4-5, L5-S1 disc herniation, and secondary spinal stenosis. ( $\mathrm{g}$ h) Show the X-ray photography of lumbar vertebra one week after surgery, promoting that the lumbar physiological curvature and the height of lumbar intervertebral space have recovered, and the internal fixation position was good.

3 months after surgery, the wound was red and swollen(Fig.1), with partial dehiscence and exudation of purulent secretion. (i j ) Show the anteroposterior and lateral radiographs of the lumbar spine five months after surgery. The results showed that the bone of the intervertebral space of L2-3, L4-5 and L5-S1 was destroyed, the gap became narrow, the edge of final plate was blurred, and the pedicle screw was loose. (k g m)Show MRI examination of lumbar spine three months after surgery. The abnormal signals of TW2 mixing, low TWI and high fat suppression image in lumbar operation area were displayed. The patients were considered to be suppurative infection after operation and underwent debridement, irrigation and drainage. In lumbar suppurative spondylitis (L2-S1), the pus culture showed Staphylococcus epidermidis. Six months after surgery, the infection was not cured. Debridement and internal fixation were performed again. (n o ) Show the anteroposterior and lateral radiographs of lumbar spine after removal of internal fixation device.

Fig.2 A 16-year-old male presented with low back pain and radiation-induced right lower limb pain for 3 months. Local tenderness and percussion pain of lumbar 3-4 vertebrae was examined at admission. WBC, ESR, C-reactive protein and calcitonin increased. (a b ) Shows the patient's anteroposterior and lateral images of the lumbar spine before operation, which shows that the lumbar 3-4 space becomes narrow and the edge of the final version is blurred. (c d e) Show the preoperative lumbar MRI examination. The abnormal signal of TW2 mixing, low TWI and high fat suppression image in front of the dural sac in the spinal canal at the posterior edge of the L3-4 space and the L3-4 space showed secondary spinal stenosis.( $f$ ) Shows the CT sagittal two-dimensional reconstruction scan of the patient's lumbar spine before operation, promoting bone destruction at the posterior and lower edge of the third lumbar vertebrae. ( $\mathrm{g} h$ ) Show the patient's lumbar anteroposterior and lateral X-ray photography one week after operation, promoting that the lumbar intervertebral space and physiological curvature partially recovered, and the position of internal fixation and bone 
grafting was satisfactory. Pathological examination showed that there were scattered acute and chronic inflammatory cell reactions in nucleus pulposus tissue and cortical bone, which was considered as suppurative spondylitis (L3-4). 


\section{Figures}
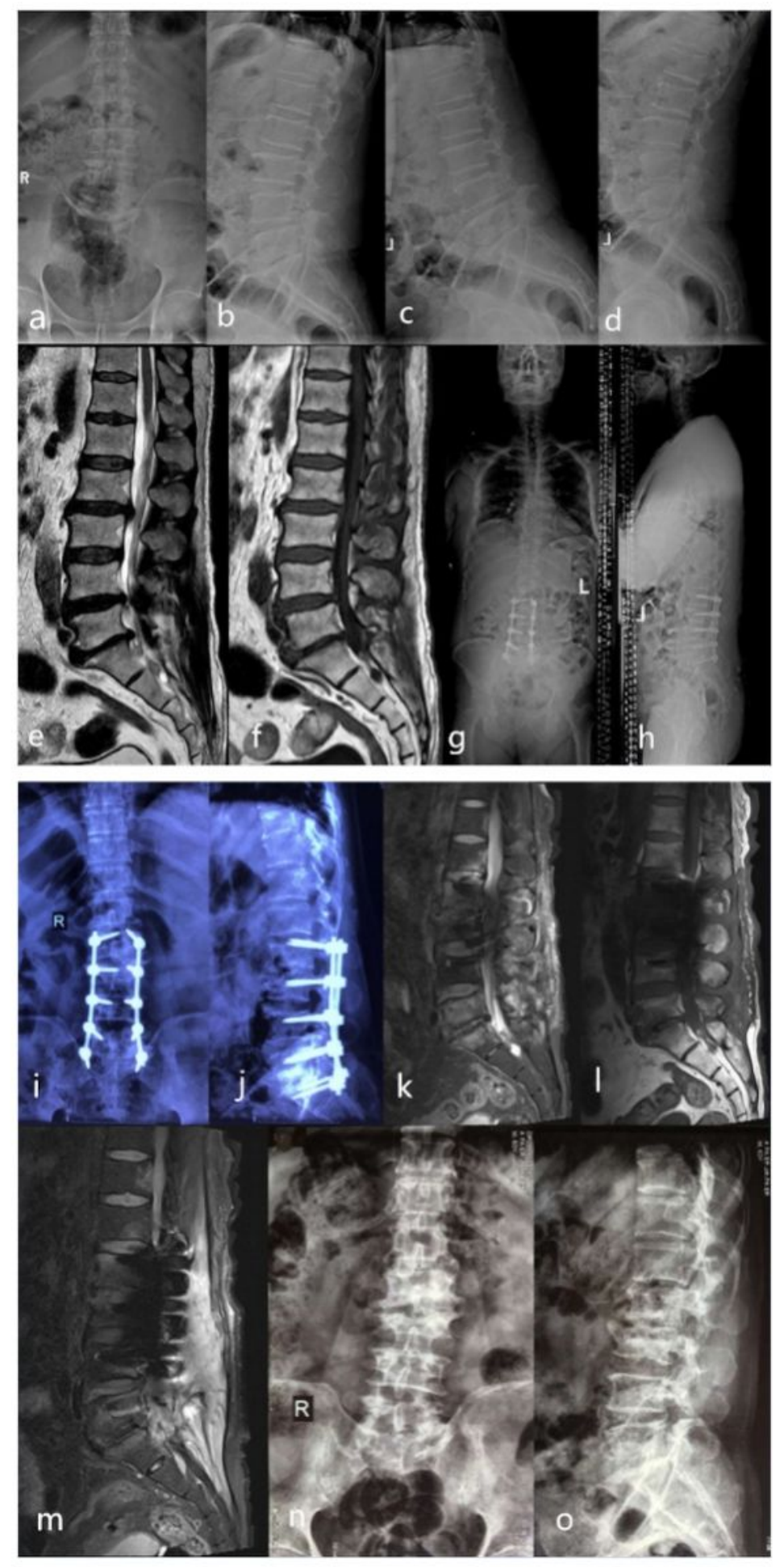

\section{Figure 1}

Male, 62 years old, (a b c d) Show the preoperative anteroposterior and lateral, flexion and hyperextension radiographs. The results showed that the physiological curvature of lumbar spine decreased, lumbar bone hyperplasia, L2-3, L4-5, L5-S1 intervertebral space narrowed, L4-5 vertebral instability. (e f) Show 
preoperative MRI of the patient, showing lumbar degenerative disease, L2-3, L4-5, L5-S1 disc herniation, and secondary spinal stenosis. $(\mathrm{g} \mathrm{h}$ ) Show the X-ray photography of lumbar vertebra one week after surgery, promoting that the lumbar physiological curvature and the height of lumbar intervertebral space have recovered, and the internal fixation position was good. 3 months after surgery, the wound was red and swollen(Fig.1), with partial dehiscence and exudation of purulent secretion. (i j ) Show the anteroposterior and lateral radiographs of the lumbar spine five months after surgery. The results showed that the bone of the intervertebral space of L2-3, L4-5 and L5-S1 was destroyed, the gap became narrow, the edge of final plate was blurred, and the pedicle screw was loose. $(\mathrm{k} \mathrm{g} \mathrm{m})$ Show MRI examination of lumbar spine three months after surgery. The abnormal signals of TW2 mixing, low TWI and high fat suppression image in lumbar operation area were displayed. The patients were considered to be suppurative infection after operation and underwent debridement, irrigation and drainage. In lumbar suppurative spondylitis (L2-S1), the pus culture showed Staphylococcus epidermidis. Six months after surgery, the infection was not cured. Debridement and internal fixation were performed again. (n o ) Show the anteroposterior and lateral radiographs of lumbar spine after removal of internal fixation device.

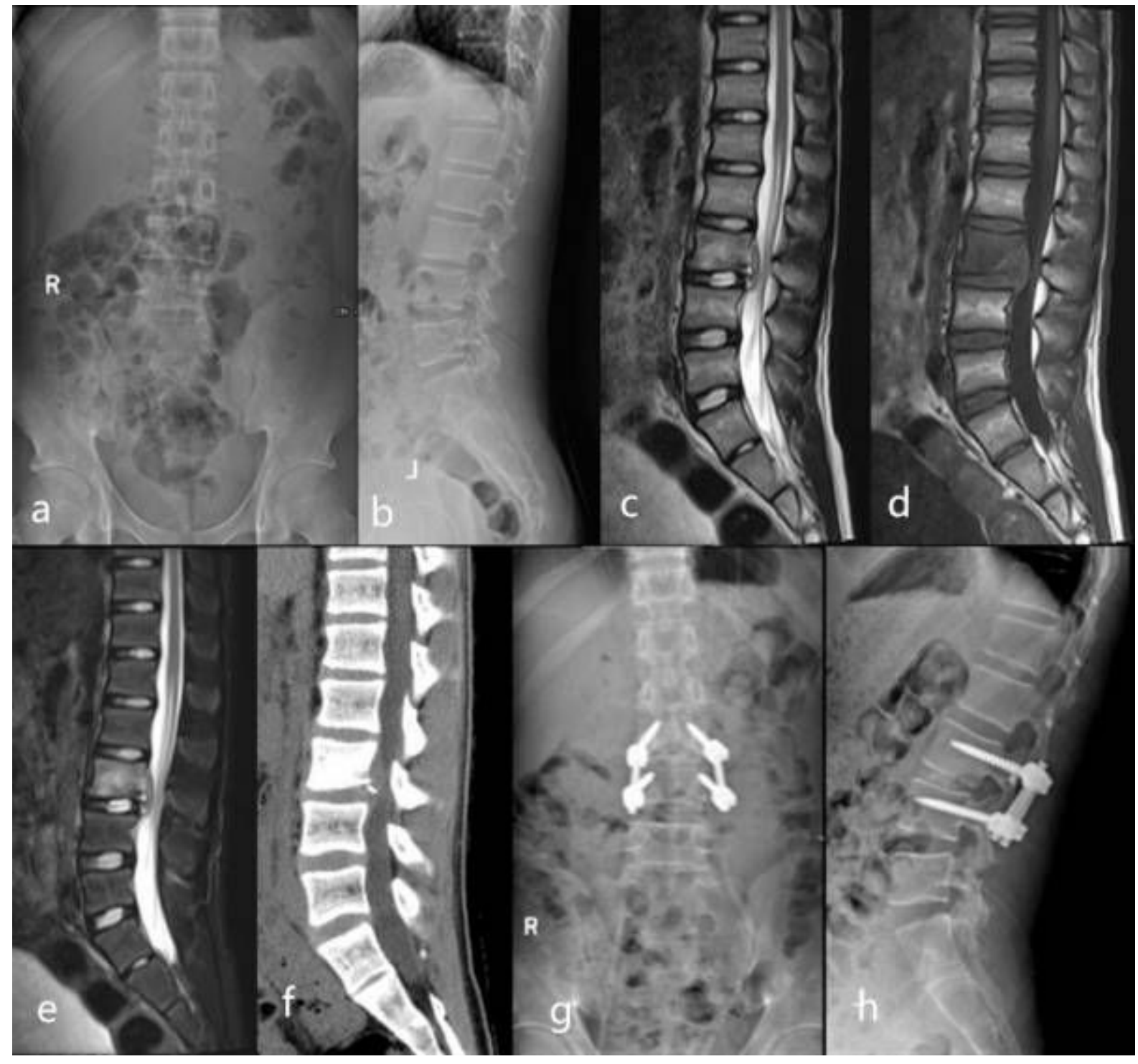

Figure 2 
A 16-year-old male presented with low back pain and radiation-induced right lower limb pain for 3 months. Local tenderness and percussion pain of lumbar 3-4 vertebrae was examined at admission. WBC, ESR, C-reactive protein and calcitonin increased. ( $a$ b ) Shows the patient's anteroposterior and lateral images of the lumbar spine before operation, which shows that the lumbar 3-4 space becomes narrow and the edge of the final version is blurred. (c d e) Show the preoperative lumbar MRI examination. The abnormal signal of TW2 mixing, low TWI and high fat suppression image in front of the dural sac in the spinal canal at the posterior edge of the L3-4 space and the L3-4 space showed secondary spinal stenosis. ( $f$ ) Shows the CT sagittal two-dimensional reconstruction scan of the patient's lumbar spine before operation, promoting bone destruction at the posterior and lower edge of the third lumbar vertebrae. ( $\mathrm{g} \mathrm{h}$ ) Show the patient's lumbar anteroposterior and lateral X-ray photography one week after operation, promoting that the lumbar intervertebral space and physiological curvature partially recovered, and the position of internal fixation and bone grafting was satisfactory. Pathological examination showed that there were scattered acute and chronic inflammatory cell reactions in nucleus pulposus tissue and cortical bone, which was considered as suppurative spondylitis (L3-4). 